\title{
The Modeling and Optimization Design of Multifunctional Movement Wheelchair Based on Ergonomics
}

\author{
TIAN Xiang Ke ${ }^{1, a^{*}}$, GUO Chun Feng ${ }^{1, b}$ and YANG Jun ${ }^{1, c}$ \\ 1 Linyi Univesity,China \\ atianxk-312@163.com, b215087595@qq.com, c116547165@qq.com
}

\begin{abstract}
Keywords: ergonomics; multifunctional movement wheelchair; modeling; optimization design Abstract. Because ordinary movement wheelchair has been unable to meet the needs of disabled sports personnel, it is necessary to research and develop multifunctional movement wheelchair. Analyzing the physical characteristics of disabled sports personnel, combining with the ideas of "human - machine - environment" system, using CATIA three-dimensional modeling software, a kind of new multifunctional sport wheelchair is designed, which conforms to the wheelchair requirements for disabled sports personnel.
\end{abstract}

\section{Introduction}

Disabled sports, such as wheelchair tennis, wheelchair basketball, wheelchair table tennis and wheelchair racing, etc., can reflect the characteristics of the disabled sports activities and make up for the defect of the disabled, which make the wheelchair sports with ordinary sports characteristics and form the the particularity of the organic combination of the wheelchair and sports for the disabled. Disabled athletes can't jump and turn like a healthy people, all participants sitting on a high sensitivity of the wheelchair, therefore performance of the wheelchair is very important for the operator. To some extent the wheelchair is athlete's "leg", the structure design, materials, and the convenience for training are different from ordinary wheelchair and has higher requirements. It is necessary to study the structure of wheelchair design and optimization[1].

\section{The Design Analysis of Multifunctional Movement Wheelchair}

The Analysis of Home work Space Design. Operation space arrangement of the movement wheelchair must follow certain principles in the design process. Work space design must begin from the requirement of man, which is basic principles to assure the safety and comfort of athletes. The position relationship between athletes and operating unit should be based on human biomechanics, the characteristics of human anatomy and physiology, following exercise principle to facilitate athletes quickly and effectively. Important and commonly used parts should be arranged in the position that the player achieve quickly and effectively. The equipment with interdependent function should be arranged together, to make athletes manipulate conveniently. Operation area should be arranged reasonably according to the order in the process of operation, making location using the body's tactile function and operating habit. In the work space design, athlete psychological impact of work space is also a factor that should be considered. Whether the Workplace design is reasonable or not, will have a direct impact on people's work efficiency [2].

Design Idea. A multifunctional sport wheelchair is designed for wheelchair sports personnel to use according to the characteristics of the wheelchair sports personnel. It should satisfy some of their special requirements.

First, the structure of the multifunctional movement wheelchair should be portable, simple and the operation should be direct, simple and easy, which can make wheelchair sports personnel enjoy the fun of the wheelchair sports truly, so as to improve the competition level of sports. Secondly, structure design of the multi-function sport wheelchair must be solid, reliable, being able to withstand large load in the process of movement, which is the most basic and stringent requirements. At the same time, the design should make consideration the feeling of athletes, for the comfort level will directly affect the athletics level of the athletes. Moreover, the conditions that threaten the safety of athletes, such as abnormal dumping, should not happen during athletic process due to the 
unreasonable design, therefore safety factors of the movement wheelchair should be considered from several aspects.

\section{The Ergonomics Research of Multifunctional Movement Wheelchair}

Ergonomics, a new edge discipline, has been developed rapidly from the 1950s. Ergonomics is a subject to study synthetically the problems such as human body structure, function, psychological, etc., using research methods and means such as anthropometry, physiology, psychology and biology mechanics and engineering, analyzing the interaction between human and machine, people and environment, and mechanical and environment, to design engineering system with the best state.

In the early stage of product design, ergonomics must be considered with the method of the system. Multi-function sport wheelchair should have a high degree of efficiency and comfort.

The system idea of "man - machine - environment" is the core to guide the overall designing scheme of the multi-function sport wheelchair. In the "human - machine - environment" system, "people" refers to the athletes, corresponding to the disabled users; "Machine" for athletes operation control object, namely multi-function sport wheelchair, "environment" corresponding assignments working space that affects driving and riding behavior.

In the design of the multifunctional movement wheelchair, ergonomics performance requirements, such as the rationality of the sitting position, ride comfort, the extension of the hands and feet, convenience of up and down the wheelchair is proposed, making "people" as the main body. The man-machine engineering design of multifunctional movement wheelchair mainly studies the interaction between the three elements of athletes, athlete operation object and working space in the system of "people - multi-function sport wheelchair - environment". For multi-function sport wheelchair research, the main content can be summarized as the following respects.

The body Characteristic Research. The main research object is issues related to the human body in the industrial design, such as the human body shape characteristic parameter, awareness and response characteristics as well as the psychological characteristics in the labor, etc. The purpose of the research is to solve how product design adapting to people's physiological and psychological characteristics, thus to create safe, comfortable and healthy and efficient working conditions for the user.

The overall Design of the Man-machine System. Whether the operating efficiency of the man-machine system is high or low depends on its overall design, that is, on the whole, make the machine adapt themselves to the human body. The basic design problem is the division of labor between man and machine, and how to effectively exchange information between people and machine, etc [3].

The Design of the Workplace and Information Transmission Device. Whether the workplace design is reasonable or not, will have a direct impact on people's work efficiency. Workplace design generally includes work space design, seat design, console design and overall layout of workplaces. The purpose of the research is to ensure that physical environment is suitable for the characteristics of the human body, making the person feel comfortable and not produce premature fatigue.

The Physiological Characte ristics of Player Sitting Position. Sitting position is the main working posture of wheelchair athletes. The incorrect posture or unreasonable seat design can make the athletes prone to fatigue, even the permanent damage to the body being unable to restore. Under the sitting driving condition, directly related to reasonable degree of the seat the affected body parts include the legs, hips, lower back and neck, etc.

The 3D Human Body Model. One of the purposes of establishing 3D human body model, is used to adapt CAD/CAM environment of the multi-function sport wheelchair design, making the internal arrangement and space design more intuitive. A more important aspect is that, can directly check the amount of space and interference using $3 \mathrm{D}$ human body model if the shape of the body surface has sufficient accuracy. The shape of the body surface must be clear, and the human body model is built on the basis of the simplified model of human body movement. CATIA software provides three kinds of display mode of the human body model, respectively expressed in branch, elliptical and human 
body surface shape, which must be able to accurately reflect the data on the human body parameters. Based on the needs of the particular working environment and efficiency, and connecting with the actual demand of the man-machine engineering evaluation, in this paper the human body model selects human body surface shape model. The virtual people of man-machine system paying attention to the movement characteristics can emulate various actions in the man-machine operation environment, to prepare for man-machine evaluation dynamically. In the process of the man-machine engineering design, people sitting in a seat of movement wheelchair, the space can make guarantee big percentile athlete body size. Hip width of the women is the design basis. To make the athletes adjust sitting position, the seat width is more than hip width[4].

\section{The Application Case of Structure Optimization Design}

Improvement to Axle tilt Way. Using two bending method can reduce the stress concentration and increase the support strength, as shown in Fig.1 and Fig.2 respectively.

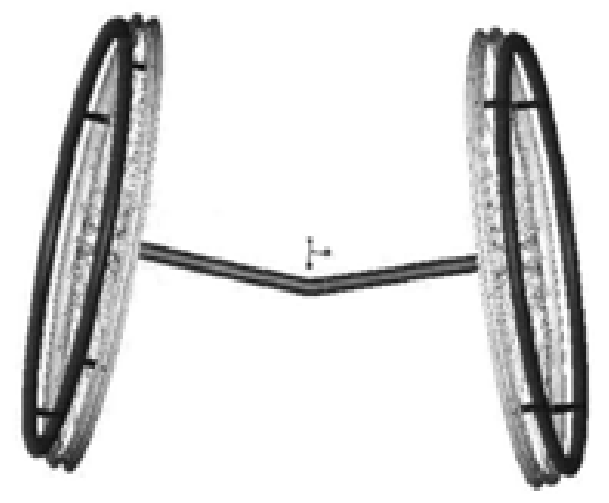

Fig. 1 Wheel axle structure before improvement

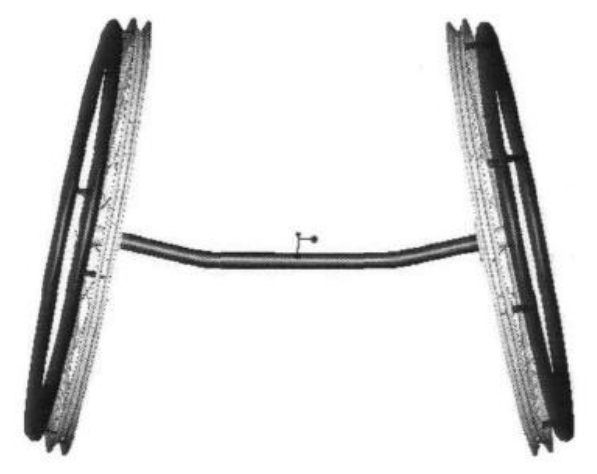

Fig. 2 Wheel axle structure after improvement

Improvement to Barycenter Position of the Body. From the point of design discipline, because the movement wheelchair needs to satisfy the motion of urgent turn, stop, barycenter of the body should be close to the central axis of the two wheels. For the existing barycenter is more close to the forward, it should be made some necessary improvements, and can be adjusted, as shown in Fig. 3 and Fig. 4.

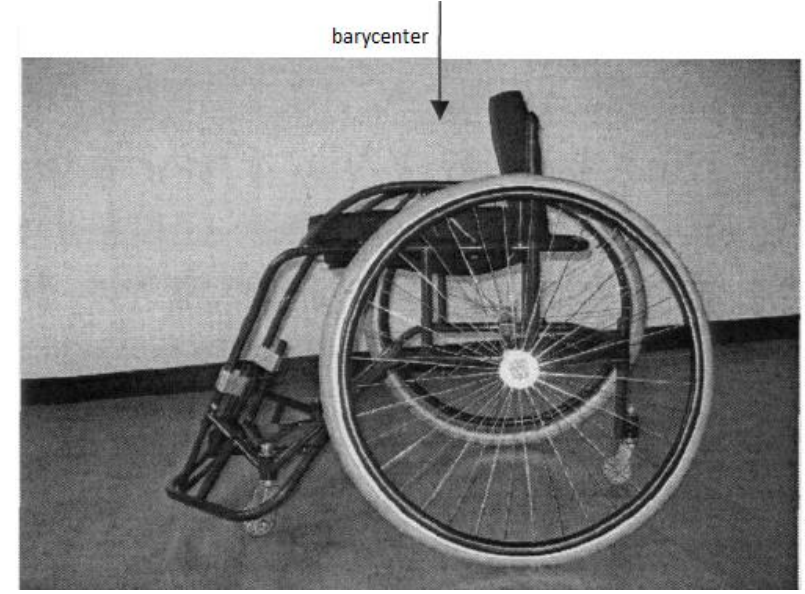

Fig.3 Barycenter position before improvement

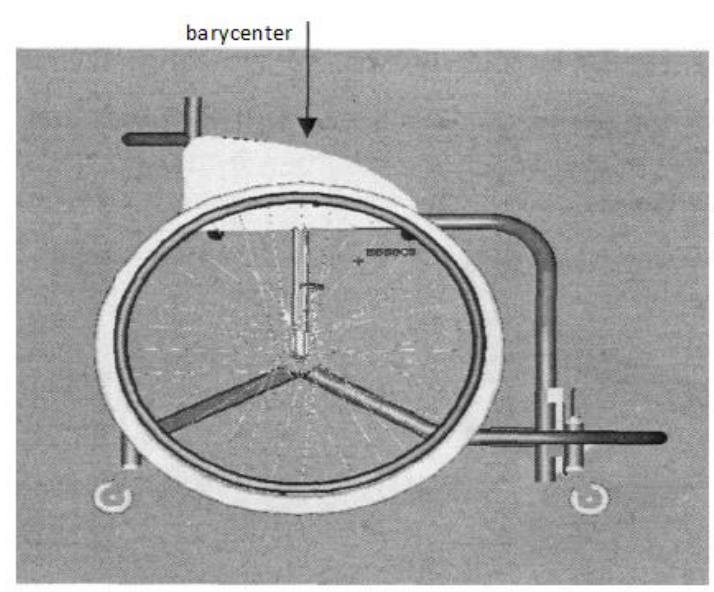

Fig.4 Barycenter position after improvement

Improvements to Anti-collision Ring. After extensive investigation and analysis, the existing anti-collision ring has many shortcomings: some anti-collision rings weld together with structural frame directly, which is not conducive to reducing vibration; Some of the anti-collision rings are too small to protect the wheelchair users; Some traditional are hard to meet the requirements of mass production and should make improvements to simplify and series; Some anti-collision rings are in the shape of line, so it is easy to appear stress concentration during molding, reducing the impact strength. In the design of new type of basketball wheelchair, between the anti-collision ring and the car body 
structure the slow connection is increased. At the same time, the size of the anti-collision ring is in accordance with the ergonomics, ensuring the safety of wheelchair users. On shape design of anti-collision ring, the shape is concise, fluent, with the transition fillet, so as to reduce the stress concentration and improve the impact strength.

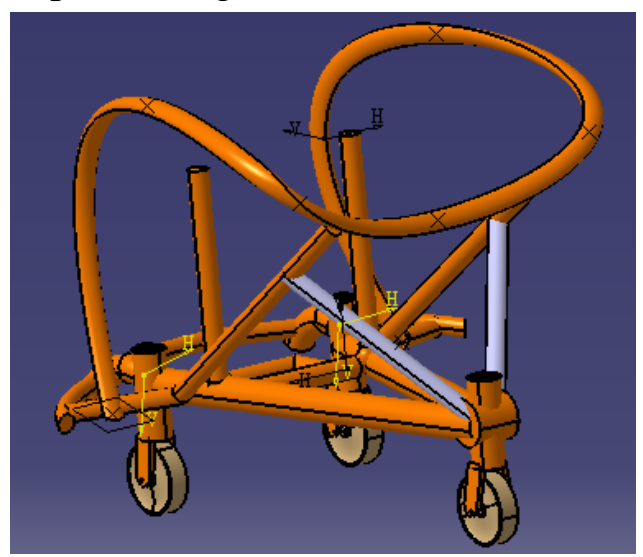

Fig.5 Design sketch of multi-function sport wheelchair

Three-dimensional Entity Modeling to Multifunctional Movement Wheelchair. In accordance with the above improvements, three-dimensional model of multifunctional movement wheelchair is established using CATIA software, as shown in Fig. 5.

\section{Conclusions}

Based on the principle of the man-machine engineering and seat design, combining the actual situation, it introduces the structural design and optimization of multifunctional movement wheelchair in the paper, breaking through single structure and function of the traditional wheelchair, increasing the humanized design, which can meet the needs of the disabled and make the competition in a more "comfortable" environment. Finally, the design was applied to the basketball sports wheelchair. With modeling platform of CATIA software, 3D digital model to the wheelchair is established. Using the virtual design method can greatly improve the design efficiency and shorten the design cycle, which can be applied to the other design of multifunctional sport wheelchair. Next work it is necessary to make motion simulation using three-dimensionaldigital model, finding out the unreasonable place, putting forward the improvement scheme. The entity prototype test and the real game simulation test need to be more discussed.

\section{ACKNOWLEDGEMENTS}

This research was funded by the project of innovative entrepreneurial training program for the college students (Grant No. 201410452025).

\section{References}

[1] LI Xia, SONG Hai-tang. The design of climbing stairs wheelchair based on ergonomics machinery.[J],2009,5:62-64

[2] FENG Yulong. Design of the basketball wheelchair based on the ergonomics [D].Tianjin: Tianjin University of Science and Technology , 2014.

[3] MA Xiao-ling, LEI Lei, GUAN Tian-min. Structure design and FE analysis of a newly basketballwheelchair. Machinery Design \& Manufacture.2008, 1:63-67

[4] LI Aiping. The Structure Design and Research of Multi-function wheelchair[D].Tianjin: Tianjin University of Science and Technology , 2010. 